\title{
Editorial
}

\section{Sidnei Quezada Meireles Leite}

Editor-Chefe

Revista Eletrônica Debates em Educação Científica e Tecnológica

Programa de Pós-graduação em Educação em Ciências e Matemática

Programa de Pós-graduação em Ensino de Humanidades

E-mail: sidneiquezada@gmail.com

Em agosto de 2011, o Programa de Pós-graduação em Educação em Ciências e Matemática (Educimat) iniciou suas atividades após aprovação da CAPES na área de Ensino (área 46) desenvolvendo estudos sobre metodologias educacionais, teorias de aprendizagem, formação de professores, uso de espaços de educação não formal, práticas pedagógicas abordando sustentabilidade, diversidades e inclusão social no contexto da educação básica, história e memórias de práticas e pessoas relacionadas ao ensino de ciências da natureza e matemática.

Já em fevereiro de 2016, imbuídos do espírito contagiante do Programa Educimat, um grupo de professores iniciou um projeto pedagógico voltado para o ensino de humanidades - nasceu o Programa de Pós-graduação em Ensino de Humanidades (PPGEH) também da área 46 da CAPES, área de Ensino. Da mesma forma que o Educimat aborda o contexto da educação científica, o PPGEH aborda estudos sobre metodologias educacionais, teorias de aprendizagem, formação de professores, uso de espaços de educação não formal, práticas pedagógicas abordando sustentabilidade, diversidades e inclusão social no contexto do ensino de história, geografia, filosofia, sociologia, artes e línguas.

A presença de alguns investigadores nas duas áreas Ensino desenvolvidas nos programa Educimat e PPGEH, produziram articulações impactaram na visão da Revista Eletrônica DECT, a qual muito gentilmente abraçou o horizonte de estudos científicos e tecnológicos no campo do ensino de humanidades. A criação desses dois programas foi uma conquista do Ifes e do Estado do Espírito Santo, para ampliar a formação de pessoas qualificadas e mentes criativas para pensar, planejar e executar projetos voltados para a educação básica e a formação de professores, sobretudo, para as regiões norte e sul do estado do Estado do Espírito Santo, norte do Rio de Janeiro, Sul da Bahia e nordeste do Estado do Minas Gerais.

O número 1 de 2016 (volume 6) da Revista Eletrônica Debates em Educação Científica e Tecnológica reúne artigos que tratam de propostas inovadoras de ensino, estudos sobre tecnologias educacionais e formação de professores no contexto da educação básica. Uma parte dos artigos desse número é remanescente da apresentação de trabalhos completos no $6^{\circ}$ Simpósio Educação e Comunicação, realizado durante o 4 - Congresso Ibero-Americano em Investigação Qualitativa, em Aracaju - Brasil, nas instalações da UTiradentes, de 5 a 7 de agosto de 2015, além de outros artigos submetidos no portal da Revista DECT em 2015. Alguns dos trabalhos foram realizados em programas de pós-graduação stricto sensu da área de Ensino e da Educação, desenvolvidos tanto em instituições nacionais como, também, em instituições internacionais.

No ano de 2016, a Revista DECT aumentou a sua periodicidade passando a ser trimestral, com números em março, junho, setembro e dezembro. Os trabalhos reúnem artigos científicos (empírico, experimental ou teórico) e ensaios, concentrados em seis eixos da revista científica, a saber: (a) Ensino de Ciências e Matemática; (b) Ensino de Humanidades; (c) Formação Inicial e Continuada de Professores da Educação Básica; (d) Tecnologias Educacionais e Recursos Didáticos; (e) Educação Profissional e Tecnológica; e (f) Diversidades e Inclusão Social. Também cadastramos a revista DECT no sistema Diadorim (Brasil) e já se encontra no banco de dados do Google Acadêmico, para melhorar o processo de busca por artigos nos aplicativos como Google, Bing e Yahoo.

Agradecemos a colaboração do Comitê Científico e dos Consultores ad hoc que dedicaram um pouco de seu tempo na avaliação dos artigos. Esperamos que os leitores aproveitem este número da Revista Eletrônica Debates em Educação Científica e Tecnológica.

\section{Equipe Editorial}

\section{Editor Chefe}

Sidnei Quezada Meireles Leite, Ifes, Brasil

\section{Comitê Executivo}

Carlos Roberto Pires Campos, Ifes, Brasil Antônio Donizetti Sgarbi, Ifes, Brasil Helio Rosetti Junior, Ifes, Brasil

\section{Comitê Científico}

Alexandre Lopes de Oliveira, IFRJ, Brasil Alexandre Maia do Bonfim, IFRJ, Brasil Álvaro Chrispino, CEFET - RJ, Brasil Antônio Henrique Pinto, Ifes, Brasil António Pedro D. Costa, UAveiro, Portugal Atanasio Alves do Amaral, Ifes, Brasil Cristiane Porto, UTiradentes - SE, Brasil Deise Miranda Viana, UFRJ, Brasil Edmar Reis Thiengo, Ifes

Eduardo Moscon, UFES, Brasil Emmanuel Favre-Nicolin, Ifes, Brasil
Evelyse dos Santos Lemos, Fiocruz, Brasil Giselle Roças de Fonseca, IFRJ, Brasil Jorge Luiz Lemos, CEFET - RJ, Brasil Jose Abdalla Helayel-Neto, CBPF, Brasil José Carlos Leivas, UFSM- RS, Brasil Katia Silva, UCDom Bosco - MS, Brasil Ligia Arantes Sad, Ifes, Brasil Luciano Toledo, IFB, Brasil Marcia Cyrino, UEL, Brasil Marco Antônio Costa, Fiocruz, Brasil Marco Braga, CEFET - RJ, Brasil Maria Alice Veiga Souza, Ifes, Brasil Maria Auxiliadora Vilela Paiva, Ifes, Brasil Mirian do Amaral Jonis Silva, UFES, Brasil Moisés Alves de Oliveira, Universidade Estadual de Londrina, Brasil Nilton Cometti, Ifes, Brasil Priscila Chisté, Ifes, Brasil Rodolfo Chaves, Ifes, Brasil Ronaldo Linhares, UTiradentes - SE, Brasil
Rony C. O. Freitas, Ifes, Brasil Rute Borba, UFPE, Brasil Sandra A. Fraga da Silva, Ifes, Brasil Sérgio Bisch, UFES, Brasil Simone de Lucena Ferreira, UFS, Brasil Susana Oliveira e Sá, UMinho, Portugal Tânia Goldbach, IFRJ, Brasil Tânia Regina Vieira, IFGoiano, Brasil Wania Gonzalez, UERJ, Brasil

\section{Comitê Técnico}

Yuri Paris Fonseca, Ifes, Brasil

Marize Lyra Silva Passos, Ifes - Ifes, Brasil

\section{Consultores Ad Hoc}

Adson Santos Bastos

Albino Oliveira Nunes

Alessandra Jordão Bicalho

Alex Jordane

Alexandre Lopes de Oliveira 
Alexandre Maia do Bonfim Alvarito Mendes Filho

Álvaro Chrispino

Amélia Rute Santos

Ana Carolina Costa Pereira

André Bernardi Candeia

Andressa Cesana

Angela Bissoli Angela Bissoli Saleme

Anna Christina Alcoforado Corrêa

Antonio Carlos Guimarães de Queiroz

Antonio Donizetti Sgarbi

Antonio Henrique Pinto

António Pedro Dias da Costa

Ardalla Ziembovicz Vieira

Atanasio Alves do Amaral

Ataualpa Oliveira

Bea Karla Teixeira

Beatriz Alves Silva

Beatriz Muller

Carina Gomes Messias Alves

Carlos Moacir Colodete

Carlos Roberto Pires Campos

Carolina Heyse Niebisch

Christine Sertã Costa

Clara Vasconcelos

Claudionor de Oliveira Pastana

Clovis Lisboa dos Santos Júnior

Cristiane Ramos Teixeira

Cristianni Antunes Leal

Daniel Landi

Danielli Veiga Carneiro Sondermann

David Paolini Develly

Deise Aparecida Peralta

Deise Miranda Viana

Diego Machado Ozelame

Diemerson Saquetto

Dilza Coco

Edmar Reis Thiengo

Edson Alkimin

Eduardo Moscon

Eduardo Vianna Gaudio

Edvonete Souza de Alencar

Eliane Aparecida Martins de Almeida

Eliza Eliza Adriana Sheuer Nantes

Elson Barbosa Silva Júnior

Emerson N. C. Gonçalves

Eugeniano Brito Martins

Evelyse dos Santos Lemos

Fabiana Carvalho Rodrigues

Felipe Oliveira Souza

Fernanda Carini

Fernanda Paixão de Souza Gouveia

Fernando Lopes

Francisco Bezerra de Lima Junior

Francisco Regis

Geisy Anny Venâncio

Gelson Vanderlei Weschenfelder

Geraldo Bull da Silva Junior

Giselle Roças de Fonseca

Glória Maria de Farias Viegas Aquije

Gustavo Cunha de Araújo

Gustavo Perini Amaral

Hamilton Viana Chaves

Helenadja Santos Mota

Hélio Rosetti Junior

Isabel Cristina Adão

Isaura Martins Nobre

Janio Alves Hilario

Janivaldo Pacheco Cordeiro

Jaqueline Ferreira Almeida

Jaqueline Maissiat

Joao Carlos Pereira de Moraes

João Henrique Viana Sousa

Jocilene Gadioli de Oliveira

Joelma Goldner Kruger

Jorge Armindo Sell
Jorge Luiz Silva de Lemos

Jose Abdalla Helayel-Neto

José Carlos Leivas

José Carlos Pinto Leivas

José Carlos Thompson

José Euzebio Simões Neto

Julia Schaetzle Wrobel

Juliana Abrão Castilho

Juliana Abrão da Silva Castilho

Juliana Santiago da Silva

Junia Freguglia

Kátia Calligaris Rodrigues

Katiuscia Viana Rocha

Katy Kênio Ribeiro

Kelly Maria Loureiro Godinho

Kleber Roldi

Larissa Merizio de Carvalho

Leivisgton Jansen Silvestre Leitao

Leonardo Polese Alves

Leonardo Salvalaio Muline

Ligia Arantes Sad

Luciane da Silva Lima Vieira

Luciano Lessa Lorenzoni

Luciano Toledo

Ludmila Pereira Rocha

Luz Marina de Souza

Magda Medhat Pechliye

Manuella Candéo

Manuella Villar Amado

Marcela Loures

Marcelo Souza Motta

Marcia Cristina da Costa Trindade Cyrino

Márcia Gonçalves de Oliveira

Marcia Regina Santana Pereira

Marciano Almeida Vieira

Marcio Alessandro Caniçali

Marco Antônio Ferreira da Costa

Marco Braga

Marco Dantas Lima

Marcus Vinicius Sandoval Paixão

Maria Alice Veiga Ferreira de Souza

Maria Auxiliadora Vilela Paiva

Maria da Gloria Médici de Oliveira

Maria das Graças Ferreira Lobino

Maria José Herculano Macedo

Maria Luiza Marques

Marize Lyra Silva Passos

Mauro Britto Cunha

Michele Waltz Comarú

Mirian Angeli

Mirian do Amaral Jonis Silva

Moacyr Cerqueira Calado

Moisés Alves de Oliveira

Nádia Ribeiro Amorim

Naíma Soltau Ferrão

Nardely Gomes

Nathalia Maria de Amorim

Organdi Mongin Rovetta

Oscar Luiz Teixeira Rezende

Osinéia Albina Brunelli

Patricia Ottz

Priscila Chisté

Raqueline Brito dos Santos

Rivana Souza Batista

Roberto Ribeiro da Silva

Robson da Silva Teixeira

Robson Vinicius Cordeiro

Rodolfo Chaves

Ronaldo Santos Santana

Rones de Deus Paranhos

Rony C. O. Freitas

Rosana M. Luvezute Kripka

Ruberley Rodrigues Souza

Rubia Carla Pereira

Rute Borba

Rutinelli da Penha Fávero
Sabrine Costa Oliveira

Sandra Aparecida Fraga da Silva

Sandra Farias Maia-Vascocelos

Sani de Carvalho Rutz da Silva

Sebastião Rodrigues Moura

Sérgio Bisch

Sidnei Quezada Meireles Leite

Sidney de Oliveira

Simone de Melo Sessa

Sonia Cristina Vermelho

Susana Emília Vaz de Oliveira e Sá

Tania Araújo-Jorge

Tânia Goldbach

Tânia Regina Vieira

Tatiana Galieta

Thamires Belo de Jesus

Thiago Marques Zanon Jacomino

Vanessa Battestin Nunes

Vargas Elenilson de Vargas Fortes

Vicente Zatti Vicente Zatti

Vilma Bragas Oliveira

Vilma Reis Terra

Vilma Reis Terra

Wallace Pereira Sant'Ana

Wania Gonzalez

Wellington dos Santos

Wesley Barbosa Rodrigues

Wesley Dondoni Colombo

William C. Smith

Willians Lopes Almeida 